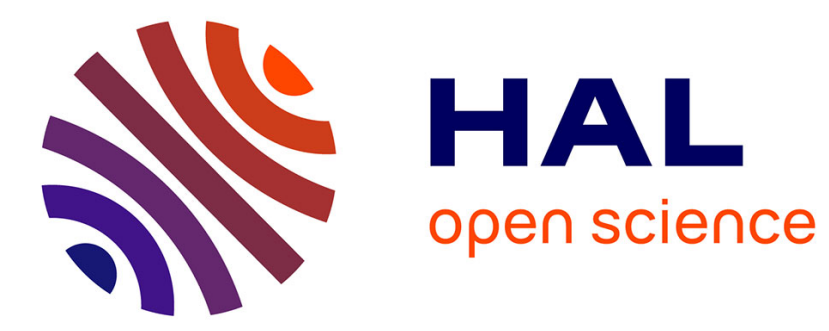

\title{
La politique étrangère du Japon depuis Meiji : essai d'interprétation
}

Jean-Marie Bouissou

\section{To cite this version:}

Jean-Marie Bouissou. La politique étrangère du Japon depuis Meiji : essai d'interprétation. Politique étrangère, 2000, 65 (3-4), pp.787-798. 10.3406/polit.2000.4983 . hal-01011198

\section{HAL Id: hal-01011198 \\ https://hal-sciencespo.archives-ouvertes.fr/hal-01011198}

Submitted on 23 Jun 2014

HAL is a multi-disciplinary open access archive for the deposit and dissemination of scientific research documents, whether they are published or not. The documents may come from teaching and research institutions in France or abroad, or from public or private research centers.
L'archive ouverte pluridisciplinaire HAL, est destinée au dépôt et à la diffusion de documents scientifiques de niveau recherche, publiés ou non, émanant des établissements d'enseignement et de recherche français ou étrangers, des laboratoires publics ou privés. 


\title{
Bouissou
}

\section{La politique étrangère du Japon depuis Meiji : essai d'interprétation \\ In: Politique étrangère N³-4 - 2000 - 65e année pp. 787-798.}

\begin{abstract}
Japan's Foreign Policy Since the Meiji Era : an Attempt of Interpretation, by Jean-Marie Bouissou Joining the modem world during the Meiji era (1868-1912), Japan has still not found its real place within the international community. At first having failed in its attempts to establish in Asia its own imperial dominion, and having still failed to transform its tremendous economie efficiency into an equivalent political power, and finally not enjoying its rehabilitation in the Asian community till the very beginning of the 1990s, Japan seems to have been endowed only recently with a global and coherent vision in foreign policy. This vision today tries to impose the image of a strong Japan, capable of bringing peace, prosperity and safety to an Asia Pacific area still in the grip of turbulences, while playing its own part in a process of globalization largely dominated by the United State.
\end{abstract}

Citer ce document / Cite this document :

Bouissou. La politique étrangère du Japon depuis Meiji : essai d'interprétation. In: Politique étrangère $N^{\circ} 3-4-2000-65 e$ année pp. 787-798.

doi : $10.3406 /$ polit.2000.4983

http://www.persee.fr/web/revues/home/prescript/article/polit_0032-342X_2000_num_65_3_4983 


\section{Jean-Marie BOUISSOU}

\section{La politique étrangère du Japon depuis Meiji : essai d'interprétation}

Entré dans la modernité avec l'ère Meiji (1868-1912), le Japon n'a peut-être pas encore trouvé sa véritable place au sein de la communauté internationale. Ayant d'abord échoué dans ses tentatives d'établir en Asie sa propre domination impériale, ayant encore échoué à transformer sa formidable puissance économique en une puissance politique équivalente et ayant, enfin, manqué sa réintégration dans la communauté asiatique jusqu'au tout début des années 90 , il semble ne s'être doté que récemment d'une vision globale et cohérente en matière de politique étrangère. Celle-ci tente d'imposer aujourd'hui l'image d'un Japon porteur de paix, de prospérité et de sécurité dans une zone Asie-Pacifique encore en proie à des turbulences, tout en jouant sa propre partition dans un processus de globalisation largement dominé par les Etats-Unis.

Politique étrangère

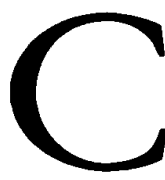

omme nous l'avons écrit ${ }^{1}$ : «Le Japon semble poser un problème de fond aux analystes des relations internationales", tant la logique qui commanderait à ses mouvements sur la scène mondiale fait l'objet des interprétations les plus opposées. Dans cet article, nous avons supposé connus les faits essenticls, pour le centrer sur trois questions : quelle a été l'efficacité de l'action extérieure du Japon de l'ère Meiji (1868-1912) à nos jours, quelles logiques a-t-clle suivies, et quelles visions la guident aujourd'hui ? Faute de place, nous nous sommes aussi permis, avec une impudeur dont nous prions le lecteur de nous excuser, de le renvoyer aux textes récents que nous avons consacrés à ces questions.

\footnotetext{
Jean-Marie Bouissou est chercheur au Centre d'études et de recherches internationales (CERI). Paris. 1. J.-M. Bouissou, "Déchiffrer "l'énigme" de la politique extérieure du Japon ". Études internationales, vol. 30 (1), mars 1999, p. 9-30
} 


\section{Efficacité...}

Au cours $d u$ siècle écoulé, le Japon semble avoir connu trois échecs majeurs dans les domaines diplomatique et militaire. Il a, en premier lieu, échoué dans ses tentatives pour établir en Asie son propre système impérialiste. Certes, il s'est d'abord insinué avec succès dans les espaces laissés libres par les Occidentaux (Ryûkyû, Formose, Corée) ; il a ensuite su jouer de leurs divisions pour avancer dans des espaces où ils étaient déjà installés (Chine) ou que certains convoitaient pour leur propre compte (Mandchourie). À ce jeu, il a gagné des concessions en Chine, des droits spéciaux en Mandchourie et les archipels enlevés aux Allemands dans le Pacifique. Mais il s'est aussi heurté aux contraintes inhérentes à son statut de "puissance régionale », obligée de limiter ses gains à ce que les grandes puissances occidentales estimaient compatibles avec leurs propres intérêts ${ }^{2}$. Contrairement au cliché d'une race "génétiquement agressive ", les dirigeants japonais ont d'ailleurs parfaitement pris en compte cette donnée. Dès le début de l'expansion coloniale, ils ont su reculer quand il le fallait (traités de Shimonoseki et de Portsmouth). En 1923, dans le cadre du «système de Washington ", ils ont accepté le gel des positions en Asie qui leur interdisait de pousser lcur avantage en Mandchourie ainsi qu'une limitation de leurs armements navals qui entérinait l'infériorité de la flotte japonaise.

On peut d'ailleurs se demander pourquoi le Japon a ensuite quitté cette ligne pragmatique pour essayer d'expulser d'Asie les grandes puissances. Les analystes ont souligné le danger qu'ont fait peser sur l'archipel les effets sociaux de la modernisation (poussée démographique, dépossession de la paysannerie) exacerbés par la crise de 1929. À côté de cette explication de la colonisation comme exutoirc aux pressions sociales et démographiques, il faut aussi évoquer l'effacement progressif de la génération qui avait connu le « pays fermé » ${ }^{3}$ et guidé ses premiers pas sur la scène internationale. À la fin des années 20 , cette génération est progressivement remplacéc par une autre, née après l'ouverture de l'archipel, comme le général en chef

2. Sur le corkept de "puissance régionale " et sor application au cas du Japon, voir S. Takahashi. "Le Japon dans l'ordre mondial. Une position perpetuellement precaire ". Etudes internationales, op. cit. [1]. p. $31 \cdot 44$

3. Sakoku: de 16335 a 1853, l'archupel n'entretient plus, en principes. d'interaction avec les monde exterieur. 
Tôjô Hideki (1884-1948). Ce dernier n'a pas connu l'angoisse extrême provoquée par l'irruption des Occidentaux et par leur formidable supériorité technologique. Mais ce sont les dirigeants modérés euxmêmes qui ont forgé, avec le "système impérial " (tennôsei), le sentiment de supériorité raciale qui a dérapé en xénophobie agressive chez la nouvelle vague de jeunes activistes sur laquelle Tôjô a surfé vers le pouvoir. Les dirigeants pragmatiques ont utilisé le tennôsei afin d'activer la transition politique entre féodalité et État moderne et de faire du Japon une nation capable de trouver l'énergie collective pour ne pas se laisser submerger par l'impérialisme occidental. Mais, la politique extérieure a ensuite subi l'effet boomerang de cette stratégie discursive développée à usage politique interne et qui a échappé à ses manipulateurs. Nous verrons que ce cas de figure - Machiavel pris au piège de sa création - semble se retrouver après la défaite avec l'image $\mathrm{du}$ «peace loving people».

Finalement, et quel que soit l'enchevêtrement des causalités, la tentative de s'affranchir du statut de puissance régionale aboutit à un échec catastrophique. Cet échec imposa des limitations draconiennes des moyens pour l'action extérieure du Japon : le fameux article 9 de la Constitution de 1947, mais aussi la division profonde de l'opinion et des élites sur les objectifs et les stratégies. La politique extérieure est alors devenue un sujet central de l'opposition entre le Parti libéraldémocrate (PLD) et la gauche. En 1960, dans une période de tensions sociales exacerbées, c'est le traité de sécurité avec les États-Unis qui déclenche la plus violente crise politique de l'après-guerre. Cette crise se dénoue par un accord implicite : d'un côté, le Parti socialiste se rallie de facto au statu quo social, mais se réserve d'utiliser un discours pacifiste et anti-américain pour rallier son électorat; de l'autre, le PLD achète la paix politique en entrant dans le jeu, observant scrupuleusement les limites à ne pas franchir dans le domaine des équipements et des missions des forces dites «d'autodéfense " - quitte, comme nous le verrons, à rajouter contrainte sur contrainte. Ainsi la politique extérieure se trouve-t-elle "prise en otage » par la politique intéricure.

Dans ces conditions, il peut sembler inévitable que le Japon n'ait pas réussi à convertir sa puissance économique en puissance politique, ce qui constituerait son deuxième échec après s'être hissé au rang de seconde économie et de premier créancier mondial (du moins jusqu'à 
la crise qui le frappe depuis 1990). On en voit souvent la preuve dans l'incapacité de la diplomatie japonaise à faire progresser d'un seul pouce ses objectifs les plus fortement proclamés : l'obtention d'un siège permanent au Conseil de sécurité de l'ONU et la récupération des îles Kouriles - alors que, dans le cas des Kouriles, le Japon jouit d'une position très forte au regard de l'histoire et du droit, d'une supériorité économique écrasante sur son adversaire et du soutien de tous ses partenaires occidentaux. On doit ajouter que Tokyo s'est trouvé, en plusieurs occasions récentes, contraint de payer pour des politiques décidées par d'autres et qui ne semblaient pas servir ses intérêts. Le cas d'école est la guerre du Golfe, que Tokyo a financée sous diverses formes pour 13 milliards de dollars, alors qu'elle ruinait vingt années d'une «diplomatie des ressources " visant à établir des liens privilégiés avec les producteurs de pétrole (dont l'Irak) qui n'étaient pas sous la coupe des majors américaines. En outre, dans le cas du programme KEDO, négocié entre Washington et Pékin pour mettre fin à la crise de 1994 dans la péninsule coréenne, le Japon ne fut guère convié qu'à le financer, alors qu'il se trouvait en première ligne comme le montra le survol de l'archipel par un missile nordcoréen en 1998.

Cette impuissance apparente nourrit l'image éculée du Japon "géant économique et nain politique ", abrité derrière le bouclier nucléaire américain en échange d'un suivisme servile, n'ayant en guise d'action extérieure qu'une "non-politique étrangère ${ }^{4}$ ». Pourtant, les résultats de cette stratégie apparaissent - sans ironie - « globalement positifs ». Elle a permis en effet au Japon de prospérer en paix, alors que des conflits meurtriers et qui mettent en ouvre la panoplic entière des armements non nucléaires n'ont pas cessé en Asic, depuis la conquête de la Chine par Mao jusqu'aux troubles actuels en Indonésic. Quant à la guerre du Golfe et au KEDO, avec du recul, on peut les créditer d'avoir stabilisé des situations qui, si elles avaient perduré, auraient bien davantage nui aux intérêts du Japon que les nouveaux équilibres qui ont résulté des initiatives prises sans lui... Par conséquent, nous sommes fondés à conclure «qu'on serait bien en peine de citer [...] une seule occurrence où le Japon aurait sacrifié sés intérêts vitaux $d u$ 
fait de sa supposée "subordination" à son protecteur américain", et que l'idée d'une faiblesse de son action extéricure ne se fonde pas sur l'examen des résultats obtenus mais uniquement sur "l'idée qu'il aurait pu faire mieux ", compte tenu de sa capacité économique ${ }^{5}$. Mais ce «mieux » est arbitrairement défini en fonction d'une vision sommairement « réaliste» des relations internationales, comme plus de capacité militaire, plus de contrôle politique sur son environnement régional et plus d'activisme dans les affaires du monde en général, sans se demander si sa posture effacée n'était pas celle qui permettait le mieux au Japon d'assurer sa prospérité et sa sécurité dans un environnement très dangercux.

Le troisième échec du Japon serait d'avoir manqué sa réintégration au sein de la communauté asiatique, à la différence de l'Allemagne, qui a su se faire accepter dans une position de puissance au sein de l'Union européenne. Mais cette comparaison ne prend pas en compte le contexte de guerre ouverte en Asie, qui interdisait à Washington de laisser la moindre autonomie à ses alliés. Elle fait fi, également, de l'hétérogénéité de l'Asic qui rendait impensable le moindre pas vers une construction régionale. Elle ignore, enfin, l'énorme différenticl de puissance entre le Japon et ses voisins, différentiel qui nourrissait la méfiance de ccux-ci et les incitait à garder les plaies de l'histoire ouvertes pour obtenir ce qu'ils pouvaient du géant par une stratégie de « chantage au péché originel».

Il n'en reste pas moins que les gouvernements PLD ont refusé de prononcer les deux termes «agression » et « excuses » qui auraient peutêtre permis de liquider l'héritage du passé, avec une obstination qui étonne l'Occidental désormais habitué aux exercices de repentance. Nous avons ici un nouvel exemple de l'interférence lourde de la politique intérieure sur la diplomatie japonaise. Le PLD est divisé entre «faucons» nationalistes et «colombes» pacifistes. De même qu'il s'ćtait conclu après 1960 un compromis entre le PLD et la gauche, il s'est aussi conclu un compromis au sein du PLD, aux termes duquel certaines expressions pouvaient être prononcées ("souffrances causées» et «profonds regrets») alors que d'autres demeuraient taboues.

5. J.-M. Bouissou, "Déchiffrer l'énigme... ", article cité [1], p. 14-18. 
Là encore, la nécessité de maintenir la stabilité politique qui confortait la prospérité économique aboutit à mettre la diplomatie dans une impasse.

Mais s'il y a eu «échec », il faut le mesurer à l'aune de ce que Tokyo aurait pu faire pour impulser un processus de construction régionale : rien, sans doute, tant que durait la guerre froide. Il faut aussi considérer ce qu'il a gagné par son inaction, c'est-à-dire rien moins que l'unité du parti gouvernemental qui contribuait à sa prospérité. Indiscutablement, en termes de soft power, le Japon y a laissé la légitimité indispensable à l'exercice d'un leadership. Mais il faut considérer les contraintes auxquelles il était soumis et, plus encore, la rapidité avec laquelle les choses ont évolué depuis que ces contraintes ont été levées en raison de la fin de la guerre froide et grâce au développement économique de la région ${ }^{6}$. L'importance de l'aide financière apportée par le Japon aux pays d'Asie touchés par la crise de 1997 - supérieure à celle du FMI - et les avancées lentes, mais réelles, de la coopération régionale (création du Forum régional asiatique, accords monétaires de Manille et de Chengmai) montrent que Tokyo est aujourd'hui en train de combler peu à peu son déficit de légitimité.

\section{Logiques...}

Comme nous l'avons vu, du début de l'ère Meiji à ce que les Japonais appellent "la guerre de la grande Asie orientale », leurs dirigeants ont été guidés par une logique conforme aux théories « réalistes » des relations internationales, fondées sur des rapports de forces dont la puissance militaire est l'instrument par excellence ${ }^{7}$, assortie d'une bonne capacité à apprécier leurs limites de «puissance régionale». Il en a résulté une politique efficace, qui aboutit à des gains territoriaux solides, avant que la capacité des dirigeants de Tokyo à évaluer les risques se dégrade, pour les raisons que nous avons évoquées plus haut. Mais pour l'après-guerre, la logique qui aurait guidé la politique

\footnotetext{
6. Les deux termes "agression" et "excuses " ont été prononcés par deux premiers ministres non PLD (Hosokawa en 1993 et Murayama en 1995). Une fois le processus enclenché, Obuchi (PLD) est allé jusqu'à des excuses officielles écrites à la Corée, dont le président a alors déclaré que "le chapitre était activement clos" (1998).

7. Pour des citations révélatrices, voir S. Takahashi, "Le Japon dans l'ordre mondial... ", article cité [2], p. 33 et 37-38.
} 
extérieure du Japon donne lieu à trois interprétations radicalement opposées.

Pour les uns, les dirigeants japonais ne seraient plus capables que d'une politique de faiblesse consistant à payer la protection américaine par un alignement inconditionnel sur Washington. Sans doute, pourtant, Tokyo a-t-il progressivement cherché à autonomiser sa politique extérieure. Les années 70 et 80 l'ont vu ainsi développer une «diplomatie tous azimuts» (zenhoi gaisho ou "diplomatie des ressources») qui visait à la fois à sécuriser les approvisionnements de l'archipel en diversifiant ses relations et à alléger la pression croissante exercée par Washington pour le forcer à libéraliser son économie. Le flirt sinojaponais, qui culmine en 1992 avec la visite de l'empereur Akihito à Pékin, n'en a été que l'ultime avatar. Mais ces tentatives n'ont eu finalement que de très maigres résultats. Elles n'ont créé avec Pékin ni confiance ni coopération, et ont engendré avec Moscou un antagonisme accru qui l'a poussé à renforcer ses armements en ExtrêmeOrient. Elles n'ont donc fait qu'emprisonner Tokyo dans une relation triangulaire avec Washington et Pékin, dont il constitue de surcroît le pôle le moins autonome et donc le plus susceptible d'être la victime $\mathrm{du}$ fameux dilemme : «abandonment or entrapment ${ }^{8}$ ».

Pour d'autres au contraire, la politique extérieure du Japon depuis 1945 traduit une volonté active de rétablir sa puissance par une stratégic graduelle. Dans un premier temps, l'archipel a remarquablement exploité sa position stratégique de base militaire indispensable aux forces américaines pour contenir le communisme en Asic. En échange de la permission donnée aux Américains d'utiliser sans contrainte son territoire pour mener leurs opérations en Corée puis au Viêtnam, le Japon a ainsi obtenu les moyens de rebâtir sa puissance économique, préalable nécessaire à toute prétention à la puissance politique. Il a bénéficié de transferts de technologic abondants et bon marché et d'une certaine indulgence pour ses innombrables manquements au jeu du libre marché. Quand les termes de l'équation ont été modifiés par les crises pétrolières et par la détente, Tokyo a su s'y adapter en se rapprochant de Pékin, voire de Moscou. Allié à l'extraordinaire capacité

8. J.-M. Bouissou, "Le Japon et les États-Unis: une relation à renégocier ", dans S. Bésanger et G. Schulders (dir.), Les relations internationales en Asie-Pacifique, Alban, Paris, 1998. 
d'adaptation de son appareil de production, ce jeu de balance, si hésitant qu'il ait été parfois, a permis au Japon de résister aux coups de boutoir américains sur le yen et au bras de fer permanent que Washington lui imposait sur les problèmes commerciaux. Et depuis que la fin de la guerre froide semble dévaloriser presque complètement l'archipel comme base militaire pour les forces américaines en Asie, Tokyo avance sur la voie d'un renforcement de ses forces armées qui pourrait lui permettre d'adopter une posture plus autonome visà-vis des États-Unis, se hissant ainsi au statut de partenaire. C'est précisément ce à quoi aboutit la renégociation du traité de sécurité en 1996-1997, avalisée par la Diète en 1999, et qui prévoit une participation active des forces japonaises aux côtés des Américains dans le cas de «crises » qui se produiraient «à proximité de l'archipel »- tout en laissant, par le vague de ces formules, une grande marge de manœuvre à Tokyo.

Une troisième école soutient qu'analyser la politique de Tokyo en termes de "force" ou de "faiblesse" masque le plus important, qui serait l'élaboration progressive d'une posture internationale originale par un pays forcé de s'adapter à des contraintes qu'aucune autre grande puissance n'a jamais eu à subir dans l'histoire mais disposant aussi d'atouts inédits. Pour désigner cette posture nouvelle, on a eu recours aux termes d' "État-marchand », de "puissance civile», de soft power, et récemment de network power. Même s'il s'agit là, au mieux, de concepts flous, tous reposent sur la logique «libérale » de relations internationales conçues comme un jeu à somme positive et sur l'idée qu'une évolution est en cours dans la nature de la puissance sur la scène mondiale - autant de notions qui apportent une nouvelle vision des objectifs de l'action extérieure.

\section{Visions...}

Les années d'après-guerre ont vu le Japon intérioriser profondément une image de lui-même comme "peace loving people»- au point que cette expression est devenue une composante fondamentale de l'identité collective nationale. Il en résulte une contrainte très forte sur les modalités désormais reçues comme acceptables pour son action internationale.

Cette image d'une nation que l'expérience historique aurait rendue en quelque sorte charnellement "éprise de paix " a d'abord été mise en 
avant par la gauche japonaise pour s'opposer à tout lien militaire avec les États-Unis. Mais elle fut ensuite reprise par les élites conservatrices. Celles-ci y ont été poussées par les tensions extrêmes qu'avait provoquées la politique de réarmement menée à la fin des années 50 et par la réduction constante de la marge de sécurité électorale du PLD. Cette réduction donnait une importance croissante au "vote flottant ", dont l'électorat féminin, qui forme l'essentiel, est animé d'un fort tropisme pacifiste. Les conservateurs ont donc repris à leur compte l'image des Japonais comme peace loving people. Cette expression est ainsi devenue l'objet politiquement ultracorrect d'un consensus discursif qui fait du pacifisme un élément de l'identité nationale, au même titre que ces autres vertus «typiquement japonaises » que seraient l'ardeur au travail ou l'esprit de groupe. Cette opération de récupération a permis au PLD de couper l'herbe sous le pied de l'opposition. Elle peut aussi s'analyser comme une manière de légitimer, en les intériorisant, les contraintes imposées par la défaite ${ }^{9}$. Mais elle a également mené les gouvernements conservateurs à officialiser successivement une série de contraintes qui s'ajoutent à l'article 9 : les trois principes non nucléaires, la limitation du budget militaire à $1 \%$ du PIB, l'interdiction de toute exportation de matériel et de technologic à usage militaire ou encore la non-utilisation militaire de l'espace...

La nécessité de respecter ce «politiquement correct » pacifiste devenu constitutif de l'identité nationale est une contrainte centrale qui pèse sur toute l'action extérieure du Japon dans les années 60-80. Mais c'est aussi le moyen par lequel la nation a su restaurer sa dignité collective - peu importe au prix de quelles manipulations - et donc retrouver, à terme, la conviction qu'elle avait «quelque chose à apporter au monde ", fondement indispensable pour que se développe une action extérieure soutenue par l'opinion. Pour les Français de 1789, ce quelque chose était la liberté. Pour les Japonais d'après-guerre, ce quelque chose est la paix. Si le Japon doit intervenir sur la scène mondiale, aux ycux de la majorité de la nation, ce ne peut être que comme "faiseur de paix".

La question de traduire cette vision dans la réalité n'a commencé à se poser que lorsque l'archipel eut atteint la puissance économique

9. S. Modjtaba et J.-M. Bouissou. "Le Japon et le monde : tout est à réinventer ", dans J.-M. Bouissou (dir.), L'envers du consensus. Les conflits et leur gestion dans le Japon contemporain. Presses de Sciences Po, Paris, 1997 
nécessaire. C'est en 1978 que le Japon s'engagea dans une ambitieuse politique d'aide publique au développement (APD), qui en fera le premier donateur mondial dans les années 80 . Mais les Japonais doivent constater que le monde ne les en remercie guère. Le principal récipiendaire - la Chine - considère leur aide comme le dédommagement trop longtemps retardé et toujours insuffisant des souffrances qu'ils lui ont infligées jadis. Les Occidentaux dénoncent l'APD de Tokyo comme un instrument destiné à ouvrir les marchés aux firmes japonaises, et une vague de nippophobie secoue à cette époque les ÉtatsUnis et l'Europe. À l'occasion de la guerre du Golfe, la contribution "humanitaire» de Tokyo fut dénoncée comme le prix honteux du sang que les Japonais se refusaient à verser. Au même moment, la diplomatie japonaise était prise bien contre son gré dans la querelle des «valeurs asiatiques ${ }^{10}$ » qui la plaçait en porte-à-faux entre l'Occident et un «asiatisme » agressif sur lequel Pékin jouait pour l'isoler (conférence de Bangkok, mars-avril 1993) ${ }^{11}$.

L'argent ne suffit donc plus. Le Japon doit aussi rendre son action lisible aux yeux du monde - ou, pour reprendre le concept de Zaki Laïdi, "faire sens ${ }^{12}$ ". Mais les dirigeants japonais eux-mêmes n'ont pas clairement défini ce sens. Le concept de "sécurité globale», adopté en 1980, fait du développement pour tous, de l'humanitaire, de l'écologie et de l'action culturelle les clefs d'une sécurité conçuc à l'échelle planétaire. Cette vision semble reposer sur le concept de "puissance civile». Mais comme au même moment la Chine renforce sensiblement ses moyens militaires, la doctrine a aussi prévu le renforcement des forces armées japonaises. Autre contradiction : alors que l'Asie semble promise à devenir "le pôle de la puissance mondiale au $X X I^{e}$ siècle ", selon l'expression en vogue avant la crise de 1997, le Japon veut y contribuer en promouvant la coopération régionale; mais il entend aussi garder les États-Unis dans le jeu, alors que l'ultralibéralisme de Wall Street est incompatible avec les modèles de développement mis en ouvre en Asie...

10. J.-M. Bouissou, "Penser l'Autre... ", article cité, et "Les "valeurs asiatiques" en question ". Géopolitique. $n^{\prime \prime}$ 67, juillet 1998, p. 15-22.

11. J.-M. Bouissou, "Penser l'Autre en se pensant soi-même. Le Japon face a l'Asie ", dans D. Camroux et J.-L. Domenach (dir.), La réinvention de l'Asie, Le Seuil, Paris, 1997.

12. J.-M. Bouissou, "Le Japon peut-il donner sens a l'Asie?", dans Z. Laïdi (dir.), Géopolitique du sens, Desclée de Brouwer, Paris, 1998. 
Face à ces contradictions, les Japonais oscillent aujourd'hui entre deux attitudes. D'un côté, la montée d'un néo-nationalisme multiforme, tant antichinois qu'anti-américain, qui déborde largement son cadre traditionnel (l'aile droite du PLD) pour atteindre le grand public des jeunes générations, via des manga à succès ${ }^{13}$. De l'autre, l'apparition d'un nouveau «politiquement correct " résumé par le terme kyôsei (symbiose) et l'image de la communauté internationale comme «un orchestre symphonique où chacun joue de son instrument particulier avec sa propre partition pour aboutir à une symphonie harmonieuse" - une métaphore très répanduc, reprise en 1995 par le Premier ministre Murayama lui-même. Cet « avatar $\mathrm{XXI}^{\mathrm{c}}$ siècle » du peace loving people mêle le vieux fond confucianiste (chacun à sa place), le post-modernisme (constitué par collage d'éléments hétéroclites), l'idéologie humanitaire (aide mutuelle et respect de l'Autre) et le discours mondialisant sur l'interdépendance. Par la multiplicité des usages auxquels il se prête, il semble avoir un bel avenir comme "vision" - ou plutôt «vitrine»- pour l'action internationale du Japon.

Plus concrètement, depuis que l'implosion du Parti socialiste, en 1996, a liquidé les tenants organisés du pacifisme institutionnel, l'actualité est à l'expansion des forces armées japonaises et de leurs missions dans le cadre du traité de sécurité révisé, sous couvert d'un «droit à l'autodéfense collective» que même le Parti démocrate, principale force d'opposition, accepte d'envisager. Le Japon a su profiter de la crise asiatique pour renforcer sa légitimité en prêtant abondamment à ses voisins sinistrés et pour esquisser discrètement l'organisation d'une possible zone monétaire moins soumise aux caprices du dollar. Enfin, Tokyo s'est attaché à présider les organismes internationaux voués à l'humanitaire (Ogata Sadako au HCR) et à la culture (Matsuura Kiichi à l'UNESCO $)^{14}$. «Sécuriser, autonomiser, légitimer» : c'est sans doute de cette façon qu'il est possible de définir aujourd'hui les objectifs de l'action internationale du Japon. On peut dire qu'il s'agit là du minimum commun à tous les pays aspirant à un rôle actif sur la scène mondiale. En ce sens, la politique extérieure du Japon n'a rien de cette

\footnotetext{
13. Non traduites... La plus typique est Shin gomanisumu sengen (" Le manifeste de la nouvelle pensée libre ") de Kobayashi Yoshinori, qui propage les théories révisionnistes à propos de la "guerre de la grande Asie... " et figure depuis plusieurs années dans la liste des dix premiers best-sellers au Japon.

14. Outre les deux mandats du Dr. Nakajima à la tête de l'Organisation mondiale de la santé (OMS).
} 
«énigme » que certains ont voulu y voir, ni de cette "irrationalité » (agressive) qu'on lui prête parfois. Et au regard des résultats obtenus depuis 1945 dans un environnement exceptionnellement dangereux et changeant, nous pensons qu'il faut lui reconnaître une efficacité qui ne peut pas s'expliquer par une «non-politique ». 